\title{
Modelling of Aircraft Emergency Evacuation: A Multiagent Approach
}

\author{
Minesh Poudel ${ }^{1}$ \\ ${ }^{1}$ LARA, Air Trans. Dept., \\ ENAC \\ Av. Edouard Belin, 31055 \\ 31055 Toulouse, France \\ minesh.poudel@airbus.fr
}

\author{
Rakia Kaffa-Jackou ${ }^{1,2}$ \\ ${ }^{2}$ EAMAC \\ Niamey, BP 746, Niger \\ rkaffa@eamac.ne
}

\author{
Felipe M. G. França ${ }^{3}$ \\ ${ }^{3} \mathrm{PESC} / \mathrm{COPPE}, \mathrm{UFR} \mathrm{J}$ \\ P.O. Box 68511, \\ 21941-972, RJ, Brazil \\ felipe@cos.ufrj.br
}

\author{
Félix Mora-Camino ${ }^{1,4}$ \\ ${ }^{4}$ LAAS du CNRS, DISCO \\ Group \\ 7 avenue du Colonel Roche \\ 31077 Toulouse, France \\ felix.mora@enac.fr
}

\begin{abstract}
In this communication we consider the problem of modeling a multi agents system evolving over a bi dimensional grid. Starting from an initial state, it is considered that the overall aim of the agent population is to behave so that the system reaches a desired final state in a minimum time. Each agent is characterized by motion parameters while to each cell is associated a current capacity. The application of interest in this study is relative to emergency evacuation of aircraft and the influence of cabin crew over its performance. A non-standard assignment problem is formulated so that cabin crew can be used efficiently during emergency evacuation. The solution of this problem should provide minimum time standards as well as insights for the design of personal guidance assistance in emergency situations.
\end{abstract}

\section{Categories and Subject Descriptors}

I.2.11 [DISTRIBUTED ARTIFICIAL INTELLIGENCE]: Multiagent systems; B.6.1 Design Styles: Cellular arrays and automata; I.6.5 Simulation and Modeling: model development; I.6.8 Types of Simulation: combined; G.1.6 Optimization: Stochastic programming.

\section{General Terms}

Distributed Artificial Intelligence, Modeling, Human Behavior.

\section{Keywords}

Aircraft emergency evacuation, cellular automata, multiagent systems, intelligent agents, .

\section{INTRODUCTION}

Demand for air travel has increased steadily over the last decades and the aviation Industry has forecast substantial growth, nearly the doubling of the air traffic, into the next coming decades. These forecasts have led aircraft manufacturers to design and produce light airframes capable of carrying as much as nine hundred passengers. One of the important aspects from the beginning of

Permission to make digital or hard copies of all or part of this work for personal or classroom use is granted without fee provided that copies are not made or distributed for profit or commercial advantage and that copies bear this notice and the full citation on the first page. To copy otherwise, or republish, to post on servers or to redistribute to lists, requires prior specific permission and/or a fee.

SIMUTools 2009, March 2-6, 2009, Rome, Italy.

Copyright 2009 ICST ISBN 978-963-9799-45-5. the aviation history is that the passenger safety has always been taken with high priority within the industry. Henceforth substantial improvement in the safety standards of the aviation from design prospective to better operations and maintenance procedures has been performed along the years.

However, though the rate of accident has decreased drastically in the last three decades, the percentage of passengers surviving after an accident has not decreased in comparison to the improvements achieved in other areas [3]. A survey by the European Transport Safety Council assesses that 40 percent out of the 1500 persons who die every year in aircraft accidents (around 600 passengers), die in technically "survivable" accidents. It has been shown that more than half of them die from the direct result of the impact, and the others die from fire, smoke or problems that arise during the emergency evacuation process [8].

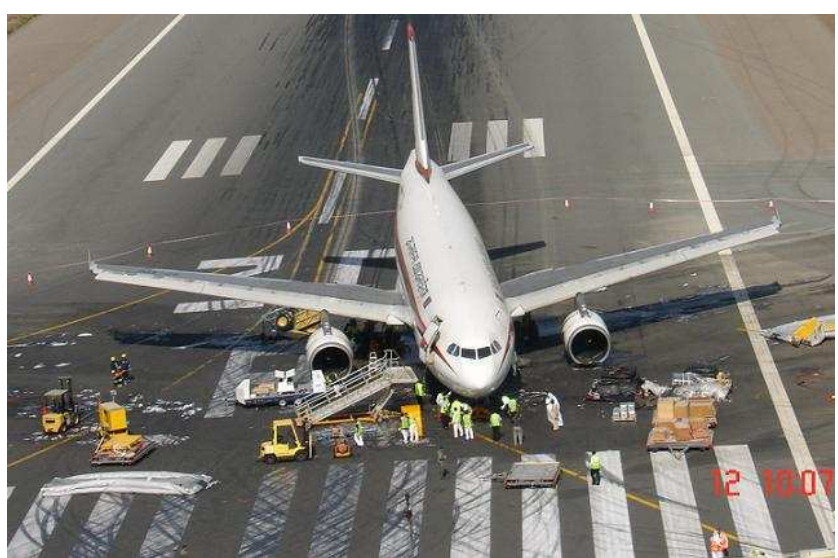

Figure 1. A310: March 07- Dubai International Airport.

Due to these reasons, not only the issues concerned with the prevention of the occurrence of accidents are tackled with great care but also issues contributing to improving the survival rate in the event of an accident/incident are of highest interest. Accidents can be classified either as fatal (non-survivable), non-fatal (survivable) or technically survivable. There are two ways to prevent fatalities in air travel: by preventing accidents and by protecting aircraft occupants when accidents occur.

In order to increase the survivability of passengers in case of an accident, one area that needs major attention is cabin safety. Cabin safety cannot be defined precisely as it covers a domain of very diverse issues such as crashworthiness, operations, human factors, psychology, and bio dynamics. However, it can be classified in 
three majors functional areas, interacting with each other namely: impact protection, fire survivability and emergency evacuation.

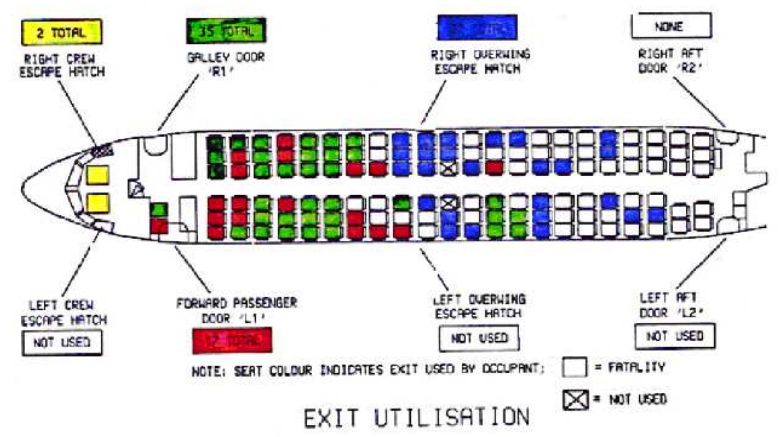

Figure 2. Exit Utilization during an accident.

The focus of the present study is on modeling emergency evacuation, which is an event which seldom occurs at the scale of daily operations by airlines and that is extremely rare at the scale of individuals. Different modeling approaches, mainly inspired on cellular automata, are reviewed [1][2][4][6][11]. Then considering that the role of cabin crew is essential for the success of emergency evacuation, a non-standard assignment problem is formulated so that cabin crew can be used efficiently during emergency evacuation. Then, it is possible to consider the behavior of egressing passengers under the optimal supervision of cabin crew. The solution of this problem should provide minimum time standards as well as insights for the design of personal guidance assistance in emergency situations.

\section{CELLULAR AUTOMATA}

Cellular Automata, which play an important role in modeling and simulation of spatiotemporal processes, appear of interest to model emergency evacuation processes [11][12]. Indeed, cellular automata are artificial mathematical models of dynamical systems, discrete in space and in time, whose behavior is completely specified in terms of some local distributed laws [9].

A typical cellular automata system is composed of four components: cells, states, neighborhood and rules. Cells are the smallest units of the system having adjoining neighbors, they are characterized by discrete states. The state of a cell can change only based on transition rules, which are defined in terms of neighborhood functions. The transition rules are the real engines of change in cellular automata. Their rules control the transformation of a cell state to another cell state over a specific period of time depending on the neighborhood of the cells. The notion of neighborhood is central to the cellular automata paradigm. Figure 3 depicts respectively the notions of neighborhood developed by von Neumann and by Moore.

An important characteristic of cellular automata is the geometry in the two-dimension space of the cells. Uniformly regular spaced square cells are used in the case of classical cellular automata. They are very often inadequate for an accurate representation of reality. To counter such situations irregular lattice structures are being introduced in the cellular automata framework.

Cellular automata have proven to be useful to analyze and understand the laws that govern complex phenomena. Cellular automata present auto organization capabilities since they can generate ordered behaviors starting from total disorder. This capability is very useful to try to explain certain kind of behaviors observed in physical, economical and biological phenomena. So, the cellular automata have been used to build numerical models of processes as diverse as chemical reactions, diffusion processes, hydrodynamic flows, mechanic, filtration and percolation. Then, computer simulations using Cellular Automata have been applied with considerable success in different areas.

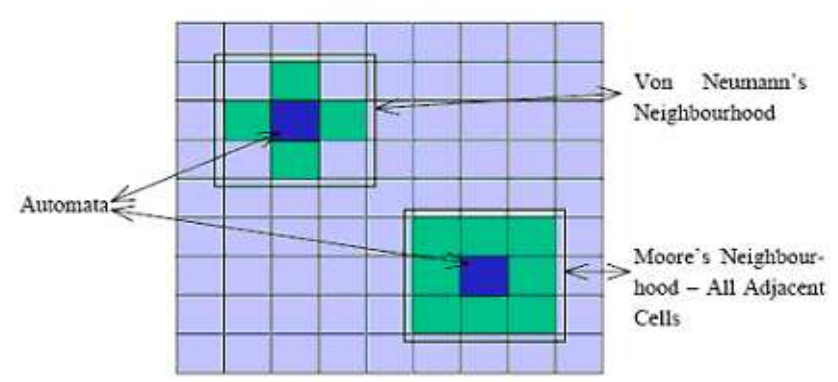

Figure 3. Cells and neighborhoods.

\section{CELLULAR AUTOMATA APPLIED TO EGRESS MODELING}

A first approach has been developed by Kirchner and Schadschneider [4] which introduced a model based on cellular automaton where space is discretized into cells (see Figure 4) which can either be empty or occupied by one person (in this case, a passenger). Each person can move to one of its unoccupied nextneighbor cells $(\mathrm{i}, j$ ) at each discrete time step $t \rightarrow t+1$ according to certain transition probabilities $p_{i j}$. These probabilities are environment dependent. A move is only possible towards one of the direct neighbor cells. For the case of the evacuation processes, the environment of a person is mainly characterized by the shortest distance to an exit door, which can be measured by the minimum number of cells that have to be crossed to reach that exit.

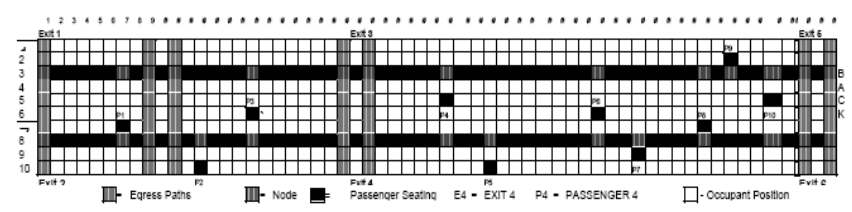

Figure 4. Discretization of aircraft cabin space.

In the model proposed by Kirchner, the passengers move from one cell to another according to rules such as:

- For a passenger, the transition probability $p_{i j}$ for a move to an unoccupied neighbor cell $(i, j)$ (including the origin cell, corresponding to no motion) is given by :

$$
p_{i j}=Q \lambda_{i j}\left(1-n_{i j}\right) e^{k_{s} s_{i j}}
$$

where $n_{i j}=0$ if cell $(i, j)$ is empty and 1 otherwise, and $\lambda_{i j}=0$ if cell $(i, j)$ is forbidden, $\lambda_{i j}=1$, otherwise. The coefficient $S_{i j}$ can be taken inversely proportional to the distance from the door measured using a Manhattan metric. Here $k_{s}$ is a positive scaling parameter and $N$ is such that:

$$
Q=\left[\sum_{i} \sum_{j} \lambda_{i j}\left(1-n_{i j}\right) e^{k_{s} S_{i j}}\right]^{-1}
$$


- Each passenger $s$ makes a probabilistic choice of a target cell according to the updated transition probability distribution $\left\{p_{i j},(i, j) \in B(s)\right\}$, where $B(s)$ is the set of direct neighbors to the cell in which passenger $s$ is currently at.

- Conflicts arising between two or more passengers attempting to move to the same cell are solved by a probabilistic method: a friction parameter $\mu \in[0,1]$ is introduced so that in a conflict the motion of all involved passengers is denied with probability $\mu$, while one passenger is allowed to move to the desired cell with probability $1-\mu$. The passenger which actually moves is chosen randomly with equal probability between the passengers involved in the same conflict.

Such a cellular automata model is unable to represent the external factors responsible for driving the dynamics of change affecting often the transition rules. To overcome such limitations, different approaches have been suggested. Among them is the integration of agent-based models over a cellular automata framework, as agent-based models can be constructed to represent the externalities driving the processes. Thus the current research is approaching towards the integration of agent-based models (multi-agent systems) with the cellular automata models, such as in the case of modeling the dynamics of emergency evacuation by incorporating different drivers as agents involved in enabling the individual spatial interactions by defining the spatial and temporal relationships to these agents.

\section{AGENT BASED MODELING}

Agents, have their origins in software engineering and artificial intelligence where they are used in networking, communications and many more applications. The aim of agent design is to create a program, which interacts with its environment. The term "agent" is usually applied to describe self-contained programs, which can control their own actions based on their perceptions of their operating environment. A significant definition is that, an agent is considered as a self-contained program capable of controlling its own decision-making and acting, based on its perception of its environment, in pursuit of one or more objectives.

Agents may be characterized by the following properties:

- clearly identifiable problem solving entities with welldefined boundaries and interfaces;

- $\quad$ situated (embedded) in a particular environment - they receive inputs related to the state of their environment through sensors and they act on the environment through effectors;

- designed to fulfill a specific purpose-they have particular objectives (goals) to achieve;

- autonomous, i.e., they have control both over their internal state and over their own behavior;

- capable of exhibiting flexible problem solving behavior in pursuit of their design objectives.

They need to be both reactive (able to respond in a timely fashion to changes that occur in their environment) and proactive (able to act in anticipation of future goals). Although, the origins of agentbased models have been in the artificial intelligence, they are also developed in the field of social sciences.

Agents can be considered as a generalization of the concept of automaton, having all features of the general automaton, with a distinction that these agents can represent the external drivers responsible for the processes. There can be as many agent-based models as the number of externalities identified driving the processes at appropriate scales. Such processes can take place at specific locations and not be system wide. While a classical cellular automata transition rule is system wide, such agent-based models would only be specific to certain locations only. These agent-based models are to act in conjunction with the regular transition rules of the cellular automata.

Multi-agent based simulation is used in a growing number of areas, where it progressively replaces the various microsimulation, object-oriented or individual-based simulation techniques, previously used. It is due, for the most part, to its ability to cope with very different models of "individuals", ranging from simple entities (usually called "reactive" agents to more complex ones ("cognitive" agents. The easiness with which modelers can also handle different levels of representation (e.g., "individuals" and "groups") within a unified conceptual framework is also particularly appreciated [2]. This versatility has made multi-agents based simulation emerge as a valuable approach for the simulation of complex systems, and it is appealing to more and more scientific domains: sociology, biology, physics, chemistry, ecology, economy, etc.

\section{MULTI-AGENTS SYSTEMS AND CELLULAR AUTOMATA}

The adoption of a multi-agent approach can be motivated by its ability to simulate autonomous individuals and the interaction between them. Agent technology is also used to simulate the outcome of the model and the simulation. Designers can use the system to assess the likely consequences of their design decisions on user behavior. The application of cellular automata implies the possibility to simulate how an 'agent'-user moves in a given environment, dependent of the behavior of other agents in the system. In developing such a simulator it is useful to differentiate between the cellular automata part and the distributed intelligence resulting of the structure of the agents, which involves the different agents with their respective roles. Various agent types may be distinguished in the model such as user-agents that represent people in the simulation. In the case of modeling emergency evacuation from aircraft, the passenger can be considered to be the subject-agent while the crew constitute the actor-agents. Thus, subject-agent and actor-agents are user-agents that navigate in the cell grid, each with their own perception, intentions and behavior. The perception of the agents is in general an imperfect representation of the virtual environment including the state of other user-agents, on which the decisions of each agent are based. Their behavior is characterized by their interaction with other agents and the environment. Different styles of behavior, like anticipated behavior and unplanned behavior, can be relevant.

Formally, user-agents can be defined by a 3-tuple $U=<R, A, F>$ where $R$ is a finite set of role identifiers, it represents the enumeration of all possible roles that can be played by user agents, $A$ represents the activity agendas of the user-agents to perform their goals and $F$ represents the knowledge or information about their environment which user agents possess. 


\section{A STOCHASTIC MULTI-AGENT \& CELLULAR AUTOMATA EGRESS MODEL}

Here is considered a system composed of $I$ subject-agents (passengers) and $J$ actor-agents (crew members) located at a grid with $N$ positions. Among these positions, a subset $N_{s}$ is composed of safe positions. The current state of passenger $i$ is represented by his position $(i(n), j(n))$ in the grid. It is assumed taht the presence of a crew member in a grid does not impair the capacity of the grid to host a passenger. It is supposed that the behaviour of egressing passengers obeys the Markov property, i.e. the imediate future can be determined with the present state information without taking into consideration information about past states. Then, the probability distribution of passengers within the grid at time $t+\Delta t, P(i=n, t+\Delta t)$, can be expressed in terms of the conditional probabilities and the previous probability distribution at time $t, P\left(i=n^{\prime}, t\right)$, which is the probability that passenger $i$ is at position $n$ ' at time $t$ :

$$
P(i=n, t+\Delta t)=\sum_{n} p\left(i=n, t+\Delta t / i=n^{\prime}, t\right) P\left(i=n^{\prime}, t\right) \quad \forall i \in L(t)
$$

where $L(t)$ is the set of alive passengers at time $t$.

Using the fact that the transition probabilities are such that:

$$
\sum_{\vec{n}} p\left(i=n, t+\Delta t / i=n^{\prime}, t\right)=1 \quad \forall i \in L(t)
$$

allows to rewrite the variation of the probability distribution as:

$$
\begin{aligned}
& P(i=n, t+\Delta t)-P(i=n, t)=\sum_{\vec{n}^{\prime}} p\left(i=n, t+\Delta t / i=n^{\prime}, t\right) P\left(i=n^{\prime}, t\right)- \\
& \sum_{\vec{n}^{1}} p\left(i=n, t+\Delta t / i=n^{\prime}, t\right) P(i=n, t)
\end{aligned}
$$

or:

$$
\begin{aligned}
& P(i=n, t+\Delta t)-P(i=n, t)=\sum_{n^{\prime}} p\left(i=n, t+\Delta t / i=n^{\prime}, t\right) \\
& \left(P\left(i=n^{\prime}, t\right)-P(i=n, t)\right)
\end{aligned}
$$

At the start of evacuation, the cabin is composed of cells, which are in the following possible states: undamaged, partly damaged but transitable, destroyed or untransitable (crushed, blasted, burnt, drowned). Possible active hazards such as fire [10], smoke and water have a starting area covering a given set of destroyed or untransitable cells. Then propagation models of the present hazards are given by:

$$
\begin{aligned}
& F(t), t \in\left\{t_{0}, t_{0}+\delta t, \cdots, t_{0}+k \delta t, \cdots, t_{f}\right\} \\
& S(t), t \in\left\{t_{0}, t_{0}+\delta t, \cdots, t_{0}+k \delta t, \cdots, t_{f}\right\} \\
& W(t), t \in\left\{t_{0}, t_{0}+\delta t, \cdots, t_{0}+k \delta t, \cdots, t_{f}\right\}
\end{aligned}
$$

where $F(t)$ is the set of cells affected by fire at period $t, S(t)$ is the set of cells affected by dense smoke at period $t$ and $W(t)$ is the set of cells affected by water at period $t$. Here $t_{0}$ is the initial time and $t_{\mathrm{f}}$ is the final period of the simulation.

Then it is possible to identify at each period the feasible exits as well as which passengers are affected by the different active disasters. Agent $i$ is alive at time $t$ if the three following conditions are met:

$$
\int_{0}^{t} \sum_{n} P(i=n, t) \varepsilon_{F, n}(t) d t \leq \sigma_{F}
$$

where

$$
\begin{gathered}
\varepsilon_{F, n}(t)=1 \text { if } n \cap F(t) \neq \varnothing \quad \varepsilon_{F, n}(t)=0 \text { if } n \cap F(t)=\varnothing \\
\int_{0}^{t} \sum_{n} P(i=n, t) \varepsilon_{S, n}(t) d t \leq \sigma_{S}
\end{gathered}
$$

where

$$
\begin{gathered}
\varepsilon_{S, n}(t)=1 \text { if } n \cap S(t) \neq \varnothing \quad \varepsilon_{S, n}(t)=0 \text { if } n \cap S(t)=\varnothing \\
\qquad \int_{0}^{t} \sum_{n} P(i=n, t) \varepsilon_{W, n}(t) d t \leq \sigma_{W}
\end{gathered}
$$

where

$$
\varepsilon_{W, n}(t)=1 \text { if } n \cap W(t) \neq \varnothing \quad \varepsilon_{W, n}(t)=0 \text { if } n \cap W(t)=\varnothing
$$

Here $\sigma_{F}, \sigma_{S}$ and $\sigma_{W}$ are positive threshold levels. It is supposed that the transition matrices are such as:

$$
P\left(i=n, t+\Delta t / i=n^{\prime}, t\right)=0 \quad \forall i \notin L(t)
$$

$$
P\left(i=n, t+\Delta t / i=n^{\prime}, t\right)=p_{n^{\prime} n}(1-\rho(n, t)) \quad \forall i \in L(t)
$$

where

$$
\rho(n, t)=\sum_{j=1}^{I} P(j=n, t)
$$

and

$$
p_{n^{\prime} n}=\alpha_{n^{\prime} n}^{*} w_{n}+\left(1-w_{n}\right) \bar{\alpha}_{n^{\prime} n}
$$

where $\alpha_{n^{\prime} n}^{*} \in\{0,1\}$ shows the best next step towards exit and $\bar{\alpha}_{n^{\prime} n} \in\{0,1\}$ is the inverse of the number of direct neighbors to cell $n$. The positive weight $w_{n}$ can be chosen such that:

$$
w_{n}=\min _{j \in C} \min _{k \in\{1, \cdots, N\}} \frac{1}{1+\lambda x_{k j}\|n-k\|}=w_{n}\left(\left[x_{k j}\right]\right)
$$

where $\lambda$ is a positive parameter and $x_{k j}=1$ if crew member number $j$ is at position $k$, and $x_{k j}=0$ otherwise and where $\|n-k\|$ is a distance on the grid of positions $n$ and $k$. The emergency evacuation can be considered completed at time $t_{f}$ when:

$t_{f}=\min t$ with $\sum_{n \in N_{s}} P(i=n, t) \geq \sigma_{i} \quad \forall i \in L(t)$

where $\sigma_{i}$ is a threshold parameter with $\sigma_{i} \in[0,1]$ but in general near to 1 . The set of safely rescued passengers, $N_{s r p}$ is such that: 


$$
\begin{gathered}
\quad i \in N_{s r p} \quad \text { if } \int_{0}^{t_{f}} \sum_{n} P(i=n, t) \varepsilon_{F, n}(t) d t \leq \sigma_{F}, \\
\int_{0}^{t_{f}} \sum_{n} P(i=n, t) \varepsilon_{S, n}(t) d t \leq \sigma_{S} \\
\text { and } \quad \int_{0}^{t_{f}} \sum_{n} P(i=n, t) \varepsilon_{W, n}(t) d t \leq \sigma_{W} \quad \forall i \in I
\end{gathered}
$$

and

\section{OPTIMIZATION OF CREW LOCATION DURING EGRESS}

Then the crew location problem during egress can be formulated as the following discrete optimization problem:

$$
\max _{\left[x_{k j}\right]}\left|N_{s r p}\right|
$$

with constraints (3), (18), (19) and the classical assignment constraints:

$$
\sum_{j \in I} x_{k j} \leq 1 \quad \forall k \in N \quad \text { and } \quad \sum_{k \in N} x_{k j}=1 \quad j \in I
$$

Of course this is a non-standard assignment problem since it includes dynamical aspects, stochastic components and dynamic opponents (hazards) [5]. An approximate solution of this problem, an heuristic based on flows in networks considerations, can be designed to tackle efficiently this problem.

\section{CONCLUSIONS}

This paper has discussed different modeling approaches that are currently used for modeling complex systems whose dynamics is characterized by the evolution, often competitive, of many individual agents: cellular automata, agent based simulation, multi-agents simulation and stochastic cell models. There, simulation is based on mathematical models that represent the temporal evolution of location of individual agents from cell to cell. It appears that all these modeling approaches present large limitations with respect to their application to emergency evacuation representation: the space in which agents move is in general composed of identical adjacent cells and cannot be easily adapted to represent realistically the confined cabin space; hazards dynamics are hardly considered; the motion of agents is driven by over simplified logics mainly based on the occupancy of neighboring cells; the behavior of the agents is assumed to be homogenous: many often there is no differentiation between the behavior of agents, no specific group behaviors are also considered. This has led to propose a stochastic model to represent egress dynamics and hazard progression as well as passengers health evolution during egress. Then an optimization problem considering the localization of cabin crew members during evacuation has been established. The aim of this problem is to locate optimally the cabin crew members so that they can provide efficient directives to evacuating passengers so that at the end of evacuation, the maximum number of life is saved.

\section{REFERENCES}

[1] Barton, J. and Paxport, L. J. 1995. Passenger and Crowd Simulation. Passenger Terminal 95 (1995),71-77.

[2] Batty, M. and Jiang, B. 1999. Multi-agent simulation: New approaches to exploring space-time dynamics within GIS. Technical Report 10, Centre for Advanced Spatial Analysis, University College London (Apr. 1999).

[3] Increasing the survival Rate in Aircraft Accidents. European Transport Safety Council (Dec. 1996).

[4] Kirchner, A. and Schadschneider, A. 2002. Simulation of evacuation processes using a bionics-inspired cellular automaton model for pedestrian dynamics. Physica A: Statistical Mechanics and its Applications 312 (Sep. 2002), 260-276.

[5] Mora-Camino, F. 2004. Modélisation, Optimisation, Complexité et Algorithmes-MOCA. Lecture Notes, CNAMToulouse.

[6] Poudel, M. and Mora-Camino, F. 2006. A Fuzzy Logic Approach for Aircraft Evacuation Modeling. In Proceedings of the International System Science Conference (Las Vegas, USA, 2006).

[7] Robson, Passenger behavior in aircraft emergencies; Technical Report No-73106. London: Royal Aircraft Establishment (1973).

[8] SFACT, Regulatory Study on Emergency Evacuations-Final Synthesis and Recommendations (Sep. 1999).

[9] Stephen, W. 1994. Cellular Automata and Complexity, Addison Wesley, USA.

[10] Takahashi, K., Tanaka, T. and Kose, T. S. 1989. An Evacuation Model For Use In Fire Safety Design Of Buildings. In Proceedings of the 2nd International Symposium on Fire Safety Science (1989), 551-560.

[11] Tissera, P.C., Printista, M., and Errecalde M. L. 2007. Evacuation Simulations using Cellular Automata. JCS\&T 7(Apr. 2007), 14-20.

[12] White, R., Engelen, G. and Uljee, I. 1997. The use of constrained cellular automata for high-resolution modeling of urban land-use dynamics. Environment and Planning B: Planning and Design 24 (1997), 323-343. 